\title{
THE KINETICS OF ERYTHROCYTE LYSIS BY ESCHERICHIA COLI HAEMOLYSIN
}

\author{
R. P. Rennie*, J. H. Freer and J. P. ARbuthnott $\dagger$ \\ Department of Microbiology, Alexander Stone Building, University of Glasgow, \\ Garscube Estate, Bearsden, Glasgow
}

\section{Plate VI}

TECHNIQUes for studying the mode of action of bacterial toxins at the molecular level are fast becoming valuable and necessary tools for the determination of the role of these agents as virulence factors in diseases of man and animals. The mechanism of action of Escherichia coli haemolysin is poorly understood. Although enough evidence now exists to conclude that calcium ions are required for haemolytic activity, only two groups of investigators have attempted to study the kinetics of erythrocyte lysis by $E$. coli $\alpha$-haemolysin.

The findings of Zwadyk and Snyder (1971) and Short and Kurtz (1971) indicated that lysis of sheep erythrocytes was dependent on haemolysin concentration, $p \mathrm{H}$ and temperature. Less haemolysis was observed as the concentration of red cells (RBC) was increased. It has been suggested that a complex consisting of haemolysin, calcium ions and RBC must be maintained until the lytic event (Short and Kurtz, 1971). These authors found that the addition of ethylenediaminetetraacetic acid (EDTA) at any time during the haemolytic reaction prevented subsequent haemolysis. They also noted that neither reducing agents nor lecithin or cholesterol affected $\alpha$-haemolysin.

Both groups of workers used methods that required centrifugation of reaction mixtures at different times, followed by spectrophotometric estimation of haemoglobin. In these processes there are unavoidable delays during which haemolysin-affected cells may be lysed.

In this study, the turbidity of reaction mixtures was monitored continuously at $650 \mathrm{~nm}$ in a controlled-temperature spectrophotometer. By this technique it was possible to make rate measurements on haemolysis curves.

\section{MATERIALS AND METHODS}

Haemolysin production and purification. The preparations of $E$. coli $\alpha$-haemolysin used in this study were produced in glucose-nutrient-broth medium and were purified by the three-stage method of Rennie and Arbuthnott (1974). Because it was more readily available, stage-II $\alpha$-haemolysin was used for most assays. However, in each experiment, purified stage-III $\alpha$-haemolysin was tested to confirm the results obtained with less pure preparations.

Received 25 Apr. 1973; accepted 6 July 1973.

* Present address: Microbiology Department, Sunnybrook Hospital, University of Toronto 2075 Bayview Avenue, Toronto, Ontario, Canada.

$\dagger$ Present address: Department of Bacteriology, University of Glasgow, Royal Infirmary, Glasgow, G4 OSF.

J. MED. MICROBIOL.-VOL. 7 (1974) 
Haemolytic assay procedure. Quantitative haemolysin titrations were performed as described previously (Rennie and Arbuthnott, 1974). Kinetic studies were carried out with silica cells of 1-cm light path in a controlled-temperature Unicam SP800 spectrophotometer, to which was attached a Unicam SP22 chart recorder (Pye-Unicam, Cambridge, England) set at a five-times multiplication factor. The spectrophotometer was adjusted so that extinction (E) was monitored at a constant wavelength of $650 \mathrm{~nm}$.

A $0.7 \%$ suspension of sheep erythrocytes (SRBC) was made in Veronal buffer, $p \mathrm{H} \mathrm{7.3}$ (Cruickshank, 1969), containing $10 \mathrm{mM} \mathrm{CaCl}$ (VC buffer). The spectrophotometer and chart recorder were then calibrated on a linear scale with the SRBC suspension. The contents of a $1-\mathrm{cm}$ cell, containing $1.4 \mathrm{ml}$ of $\mathrm{VC}$ buffer and $0.1 \mathrm{ml}$ of $0.7 \%$ SRBC, were mixed by inversion and read at $\mathrm{E}_{650}$. The zero controls of the spectrophotometer and chart recorder were adjusted if necessary such that the $\mathrm{E}_{650}$ was 0.40 . This represented $100 \%$ erythrocyte concentration. The same procedure was repeated using $0.05 \mathrm{ml}$ of $0.7 \%$ SRBC and $1.45 \mathrm{ml}$ of VC buffer. An $\mathrm{E}_{650}$ of 0.20 represented $50 \%$ erythrocyte concentration and the spectrophotometer, chart recorder and SRBC suspension were considered to be standardised. VC buffer was used in the reference cell.

In the test system, $0.1 \mathrm{ml}$ of $\alpha$-haemolysin or dilution thereof was added to $1.3 \mathrm{ml}$ of VC buffer in a spectrophotometer cell and the solution was mixed rapidly by inversion of the cell. After incubation for $1 \mathrm{~min}$. at $37^{\circ} \mathrm{C}, 0.1 \mathrm{ml}$ of standardised $0.7 \%$ SRBC was added, the test cell was again mixed by inversion and was replaced in the spectrophotometer. Lysis of erythrocytes was measured by monitoring continuously the reduction in $\mathrm{E}_{650}$. Rates of haemolysis were determined by measuring the slope over the linear portion of haemolytic reaction plots.

Buffers and chemicals. Sodium cacodylate- $\mathrm{HCl}$ buffer $(p \mathrm{H} 5.0$ to 7.4$)$ and $0.01 \mathrm{M}$ tris (hydroxymethyl) aminomethane-HCl (Tris) buffer ( $p \mathrm{H} 7 \cdot 2$ to $9 \cdot 0$ ), containing $10 \mathrm{mM} \mathrm{CaCl}$, were prepared according to the methods described by Dawson et al. (1969). The EDTA used was an analytical grade reagent (BDH, Poole) and was made up in Veronal buffer, $p H \mathbf{7} \cdot 3$, such that its final concentration in reaction mixtures was $5 \mathrm{~mm}$.

Electron microscopy. Samples of stage-III $\alpha$-haemolysin were dialysed against distilled water to remove salts. Thus prepared, $1 \mathrm{mM} \mathrm{CaCl} 2$ was added to one sample; the other was left untreated. After $10 \mathrm{~min}$. at $37^{\circ} \mathrm{C}$, portions of these samples were placed on carboncoated formavar films on copper grids. Negative staining was performed with $2 \%(\mathrm{w} / \mathrm{v})$ aqueous ammonium molybdate and the samples were examined in a Philips EM300 electron microscope operating at $60 \mathrm{kV}$.

\section{RESULTS}

The spectrophotometric assay technique was especially suited to kinetic studies for two reasons: $(a)$ continuous assessment of the haemolytic reaction was possible and $(b)$ the amount of substrate (SRBC) added in each experiment could be accurately standardised.

As shown in fig. 1, the haemolytic reaction was characterised, even with high concentrations of $\alpha$-haemolysin, by an initial lag phase followed by a period of lysis that was linear between 20 and $80 \%$ haemolysis. When the lag phase and rate of haemolysis were plotted against the logarithm of haemolysin concentration (fig. 2), it was found that the lag phase was inversely proportional to haemolysin concentration. The rate of haemolysis increased constantly with high concentrations of haemolysin but tailed off when less than $2000 \mathrm{HU}$ were added to the reaction mixture.

The rate of haemolysis was affected also by $p \mathrm{H}$ and temperature of incubation. Maximum rates were found at $p \mathrm{H} 8.8$ to 9.0 and a temperature of $43^{\circ} \mathrm{C}$. The lag phase remained constant, except in experiments performed at 


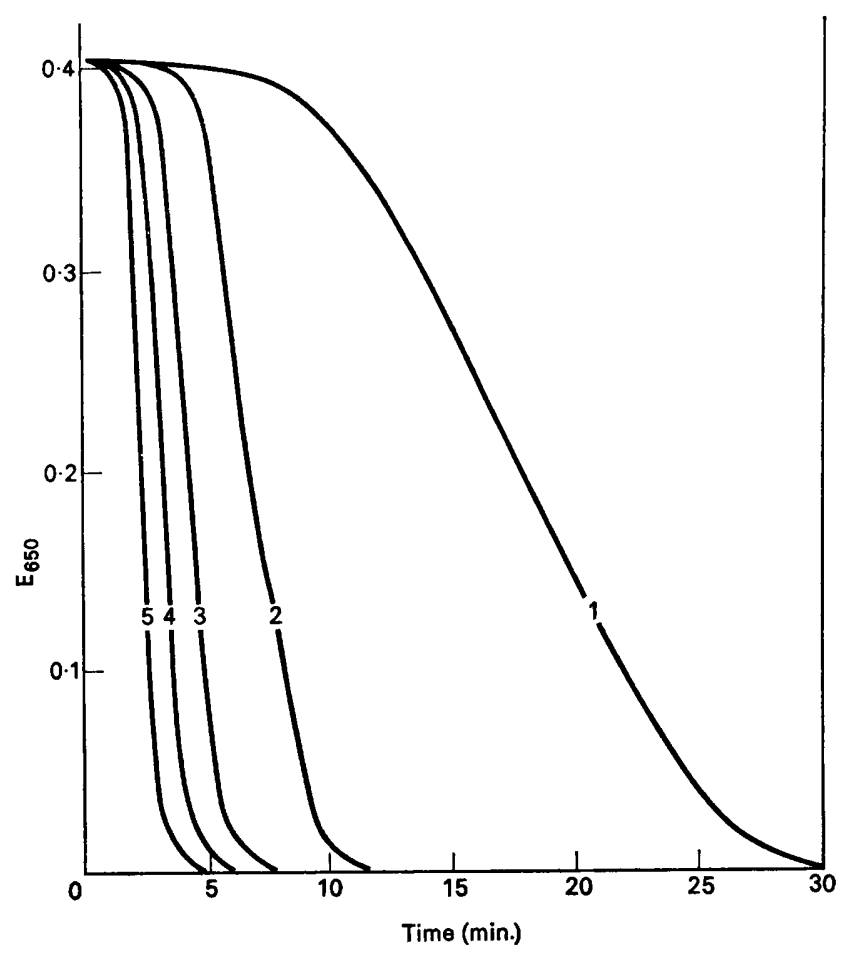

FIG. 1.-Effect of $\alpha$-haemolysin concentration on the kinetics of haemolysis. $\mathrm{CaCl}_{2}=10 \mathrm{~mm}$ (final); temperature $=37^{\circ} \mathrm{C} ; 1=1.8 \times 10^{2} \mathrm{HU} ; 2=1.8 \times 10^{3} \mathrm{HU} ; 3=1.8 \times 10^{4} \mathrm{HU} ; 4=9.0 \times 10^{4}$ $\mathrm{HU} ; 5=1.8 \times 10^{5} \mathrm{HU}$.

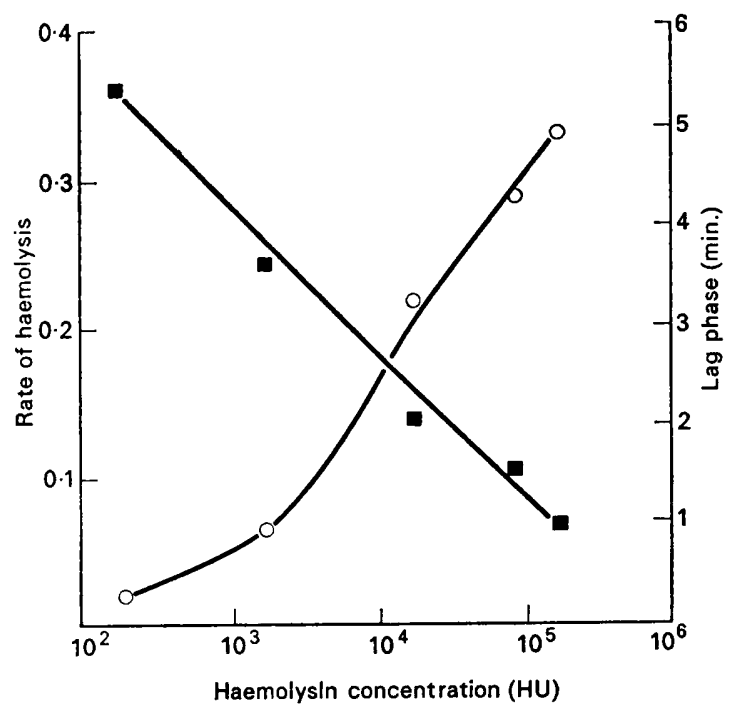

FIG. 2.-Effect of $\alpha$-haemolysin concentration on length of lag phase and rate of haemolysis. - lag phase (min.); $0-0$ rate of haemolysis at $\alpha$-haemolysin concentrations given in fig. 1 .

J. MED. MICROBIOL.-VOL. 7 (1974) 


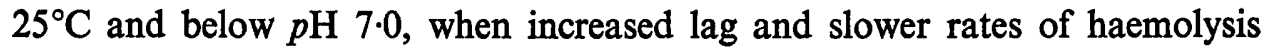
were observed.

The rate of haemolysis was not altered by concentrations of $\mathrm{CaCl}_{2}$ between $0.1 \mathrm{~mm}$ and $100 \mathrm{~mm}$ (fig. 3). However, between $1 \mathrm{mM}$ and $10 \mathrm{mM} \mathrm{CaCl}$, the length of the lag phase was reduced to a minimum. Slight haemolytic activity was noted in the absence of externally supplied calcium ions, but the lag phase was exaggerated and the rate of haemolysis was slow. Neither magnesium ions (as $10 \mathrm{~mm} \mathrm{MgSO} 4.7 \mathrm{H}_{2} \mathrm{O}$ ) nor manganese ions (as $10 \mathrm{~mm}$ $\mathrm{MnCl}_{2}$ ) could be substituted for calcium ions.

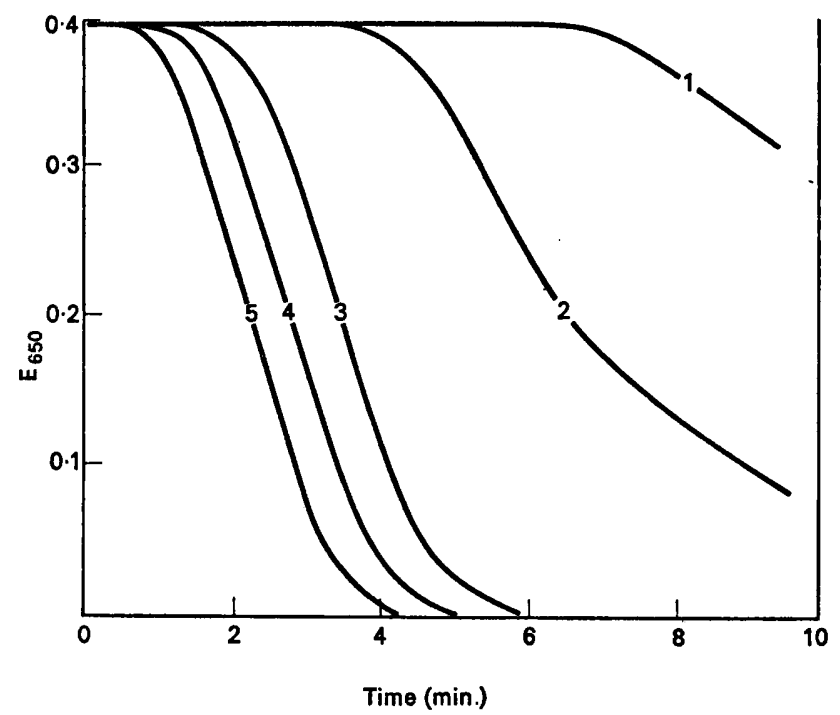

FIG. 3.-Effect of calcium-ion concentration on the kinetics of haemolysis. $\alpha$-haemolysin $=9.0 \times 10^{4}$ $\mathrm{HU}$; temperature $=37^{\circ} \mathrm{C} ; 1=$ no $\mathrm{CaCl}_{2}$ added; $2=\mathrm{CaCl}_{2}(0.01 \mathrm{~mm}$ final $) ; 3=\mathrm{CaCl}_{2}$ (100mm final); $4=\mathrm{CaCl}_{2}\left(0 \cdot 1 \mathrm{~mm}\right.$ final); $5=\mathrm{CaCl}_{2}(1,2,5$ and $10 \mathrm{~mm}$ final gave identical reaction curves).

The finding that in all experiments a lag phase occurred before erythrocyte lysis invited a more detailed investigation of this part of the haemolytic reaction. In the experiments mentioned above, $\alpha$-haemolysin was incubated in buffer containing calcium ions for 1 min. to bring the buffer and haemolysin to the temperature of the system before adding SRBC. To obtain further information about the function of calcium ions in the haemolytic reaction, $\alpha$-haemolysin was pre-incubated at $37^{\circ} \mathrm{C}$ with $10 \mathrm{mM} \mathrm{CaCl}_{2}$ for varying periods of time before the addition of SRBC. At the concentration of $\alpha$-haemolysin used (fig. 4), pre-incubation with calcium ions to a maximum of $5 \mathrm{~min}$. caused a progressive reduction in the lag phase while the rate of haemolysis was not affected.

To determine whether calcium ions were required throughout the haemolytic reaction, a series of kinetic experiments were designed in which EDTA was used as a chelating agent for calcium ions. As shown in fig. 5, additions of EDTA to the pre-incubation mixture of $\alpha$-haemolysin and $\mathrm{CaCl}_{2}$ inhibited 
haemolysis when SRBC were added 2 min. later (curve 1). However, if calcium ions were allowed to react with the haemolysin for $2 \mathrm{~min}$. before EDTA was

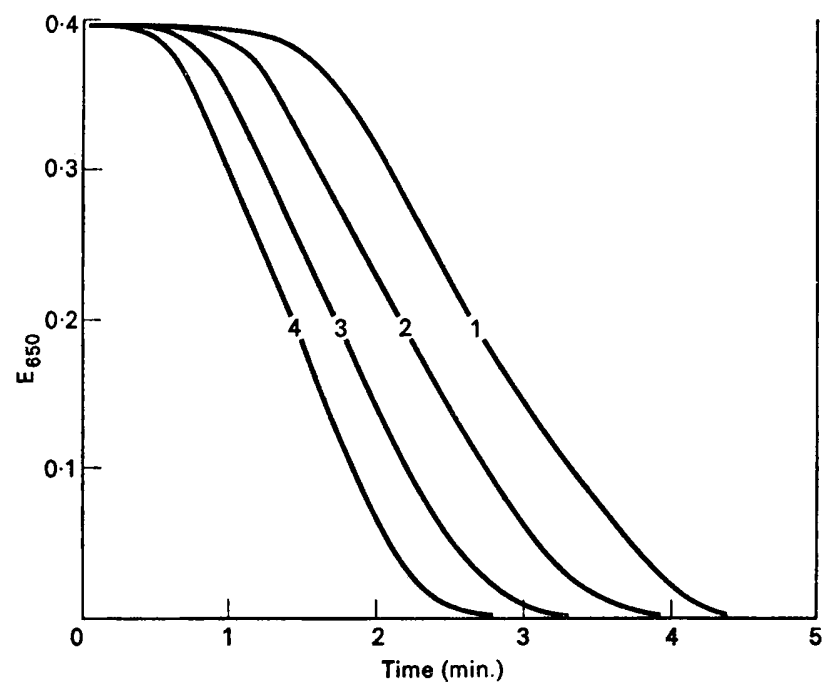

FIG. 4.-Pre-incubation of $\alpha$-haemolysin and calcium ions before initiating haemolysis by adding sheep RBC. $\alpha$-haemolysin $=9.0 \times 10^{4} \mathrm{HU} ; \mathrm{CaCl}_{2}=10 \mathrm{mM}$ (final); temperature $=37^{\circ} \mathrm{C}$; $1=$ no pre-incubation; $2=1 \mathrm{~min}$. pre-incubation; $3=2 \mathrm{~min}$. pre-incubation; $4=5$ to 20 min. pre-incubation (identical reaction curves).

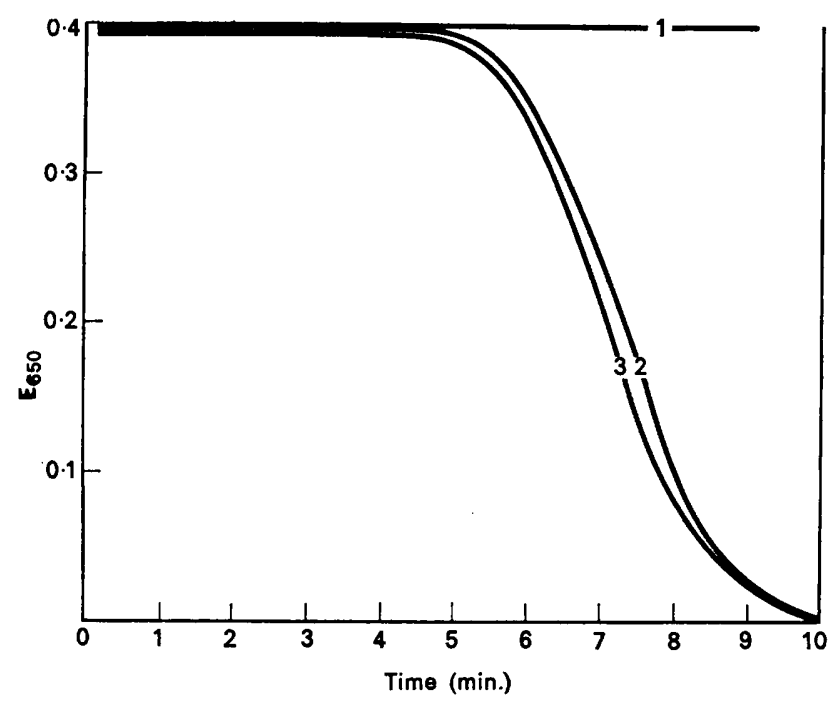

FIG. 5.-Effect of EDTA on lysis of sheep RBC by $\alpha$-haemolysin. $\alpha$-haemolysin $=3.0 \times 10^{3} \mathrm{HU}$; $\mathrm{CaCl}_{2}=2 \mathrm{mM}$ (final); EDTA $=5 \mathrm{~mm}$ (final); temperature $=37^{\circ} \mathrm{C}$. Haemolysin was incubated with $\mathrm{CaCl}_{2}$ for $0 \mathrm{~min}$. (reaction 1) and $2 \mathrm{~min}$. (reaction 2) before the addition of EDTA. No EDTA was added in reaction 3. Sheep RBC were added after $2 \mathrm{~min}$. to each reaction mixture.

added to the system (curve 2), comparison with the control reaction (curve 3) shows that inhibition of haemolysis did not occur under these conditions. EDTA by itself did not cause lysis of SRBC. In addition, when SRBC were 
removed by centrifugation from the reaction mixture $3.5 \mathrm{~min}$. after the start of the experiment and were resuspended in Veronal buffer containing EDTA but not $\mathrm{CaCl}_{2}$, we noted that 25 to $30 \%$ lysis of erythrocytes occurred. This indicated that $\alpha$-haemolysin that had been adsorbed to SRBC was not affected by EDTA.

The effect of calcium ions on purified $\alpha$-haemolysin is shown in fig. $6 a$ and $6 b$. In the absence of calcium ions (fig. 6a), $\alpha$-haemolysin appeared in the electron microscope mainly as elongated amorphous aggregates. By contrast, $\alpha$-haemolysin treated with calcium ions (fig. $6 b$ ) became orientated into regular structures with a spherical appearance. Some of these latter forms were seen in untreated preparations. Measurement of 100 of the structures seen in fig. $6 b$ gave a normal distribution with a maximum at $15 \mathrm{~nm}$, and this value was used to estimate the molecular weight of purified $\alpha$-haemolysin (Rennie and Arbuthnott, 1974).

\section{Discussion}

The aim of this study was to obtain experimental evidence about the mechanism of erythrocyte lysis by $E$. coli $\alpha$-haemolysin. As mentioned, the haemolytic reaction consisted of a lag phase and a period of lysis. By contrast, Zwadyk and Snyder (1971), by sampling at 1-min. intervals, found that high concentrations of $\alpha$-haemolysin abolished the lag phase. This disparity illustrates the importance of continuous monitoring of reactions, which allows accurate determination of rates of haemolysis to be made.

Our findings indicate that the first step in the haemolytic reaction is activation of the haemolysin by calcium ions, a process that induces a change in the structural appearance of the molecule (fig. $6 a$ and $6 b$ ). This activation was shown by a reduction in the lag phase when the pre-incubation time with $\mathrm{CaCl}_{2}$ was increased (fig. 4). Results of the experiments in which EDTA was added (fig. 5) suggest that during activation calcium ions are firmly bound to $\alpha$-haemolysin molecules. Inhibition of haemolysis occurred only when EDTA was added to $\alpha$-haemolysin before activation with calcium ions. By contrast, Short and Kurtz (1971) found that the addition of EDTA to reaction mixtures prevented haemolysis at any time during the haemolytic reaction. However, they used a different assay system; haemolysin was not pre-incubated with calcium ions in the absence of erythrocytes and they used considerably lower concentrations of $\alpha$-haemolysin. Whether these differences can explain the inhibitory effect of EDTA is uncertain.

Although the evidence presented in this investigation indicates that activation of $\alpha$-haemolysin by calcium ions is the foremost step in the haemolytic reaction, elucidation of the mechanism of erythrocyte lysis remains to be determined. The inverse proportionality of the lag phase to the logarithm of haemolysin concentration does not suggest a reversible enzymic mechanism of action. An arithmetic relationship between lag phase and haemolysin concentration would be expected in an enzymic reaction. The broad spectrum of haemolytic activity (Rennie and Arbuthnott, 1974) suggests that $\alpha$-haemolysin does not 


\section{ESCHERICHIA COLI HAEMOLYSIN KINETICS}

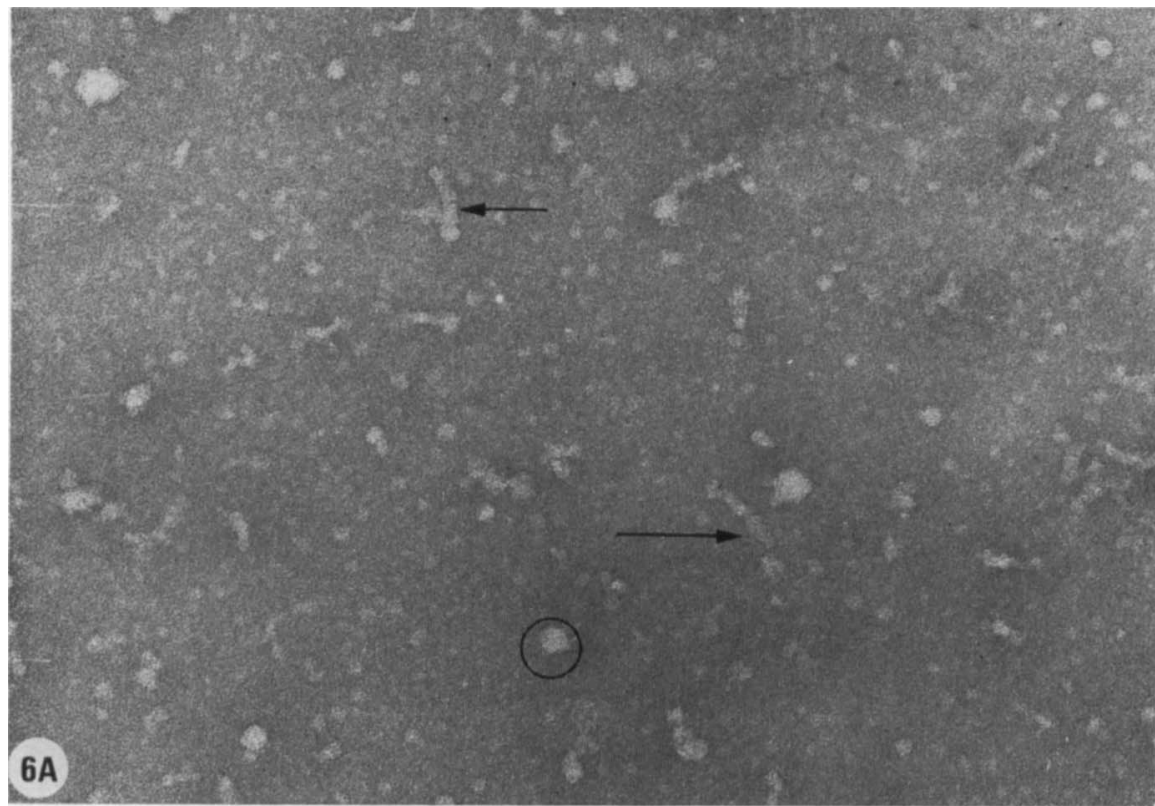

FIG. $6 a$.-Stage-III $\alpha$-haemolysin in the absence of calcium ions, negatively stained with $2 \%$ aqueous ammonium molybdate. Note the elongated aggregates (arrow) and a few spherical particles (circle). EM. $\times 145,000$.

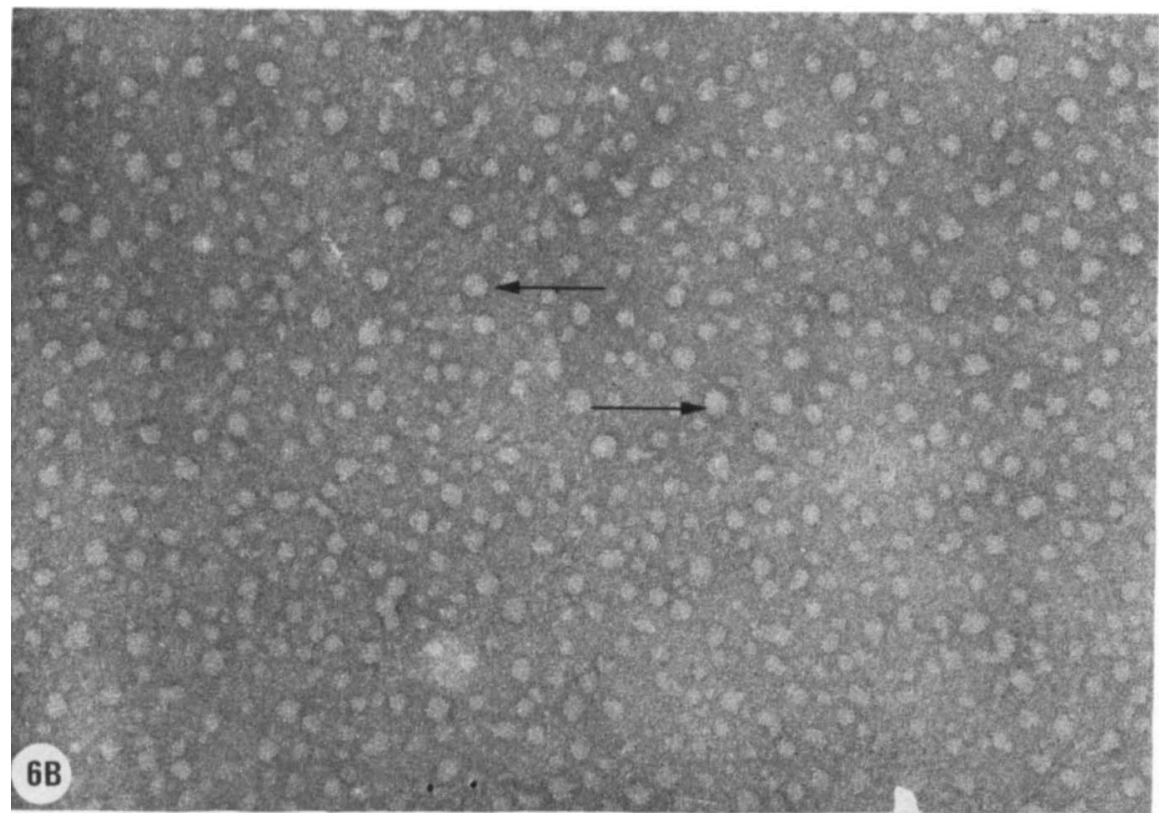

FIG. $6 b$.-Stage-III $\alpha$-haemolysin treated with $1 \mathrm{~mm} \mathrm{CaCl} 2$ for $10 \mathrm{~min}$. at $37^{\circ} \mathrm{C}$ and negatively stained with $2 \%$ aqueous ammonium molybdate. Note the large number of uniform spherical structures (arrow). EM. $\times 145,000$. 
interact with specific phospholipid residues in red-cell membranes. Also, it was found (Rennie, 1973) that water-soluble phosphorus was not released from lecithin or sphingomyelin emulsions, indicating that $\alpha$-haemolysin contains no phospholipase-C activity.

The mechanism of action of such well-studied cytolytic toxins as the streptolysins, staphylococcal $\alpha$ - and $\delta$-toxins and Clostridium perfringens $\theta$-toxin is not yet fully understood (Bernheimer, 1970), and it is to be expected that some time will elapse before the specific action of $E$. coli $\alpha$-haemolysin is elucidated. To find out whether ionic or hydrophobic forces are dominant in this haemolytic reaction, a detailed study of the interaction of the agent with red-cell ghosts and with model lipid-membrane systems will be necessary.

\section{SUMMARY}

The interaction of Escherichia coli $\alpha$-haemolysin with sheep erythrocytes was characterised in all experiments by a lag phase, and by a period of lysis that was linear between 20 and $80 \%$ haemolysis. The lag phase was inversely proportional to the logarithm of haemolysin concentration. Maximum rates of haemolysis were observed at $43^{\circ} \mathrm{C}$ and $p \mathrm{H} 8.8$ to $9 \cdot 0$.

The optimal concentration of $\mathrm{CaCl}_{2}$ for haemolysis was between $1 \mathrm{mM}$ and $10 \mathrm{~mm}$. Evidence is presented that the first step in the haemolytic reaction is activation of $\alpha$-haemolysin by calcium ions. This causes an alteration in the structural appearance of purified haemolysin molecules. It was also shown that EDTA inhibited haemolysis only when added to $\alpha$-haemolysin before its activation by calcium ions. This finding suggests that, during activation, calcium ions are firmly bound to $\alpha$-haemolysin.

The authors wish to acknowledge the co-operation of Dr T. A. McAllister, Queen Mother's Hospital, Yorkhill, Glasgow. This work was done while one of us (R. P. R.) was in receipt of a Medical Microbiology Studentship from the University of Manitoba, Canada. In part, the work was supported by grants from the Royal Society and the Scottish Home and Health Department.

\section{REFERENCES}

Bernheimer, A. W. 1970. Cytolytic toxins of bacteria. In Microbial toxins, vol. 1, Bacterial protein toxins, edited by S. J. Ajl, S. Kadis and T. C. Montie, New York and London, p. 183.

CRUICKSHANK, R. 1969. In Medical microbiology, 11 th ed., revised reprint, Edinburgh, p. 861.

Dawson, R. M. C., Elliot, D. C., Elliot, W. H. AND JoNes, K. M. 1969. In Data for biochemical research, 2nd ed., Oxford, pp. 488 and 494.

RENNIE, R. P. 1973. Haemolytic activity of Escherichia coli. PhD Thesis, University of Glasgow, p. 125.

RenNie, R. P. AND Arbuthnotr, J. P. 1974. Partial characterisation of Escherichia coli haemolysin. J. med. Microbiol., 7, 179.

Short, E. C. AND KurTz, H. J. 1971. Properties of the hemolytic activities of Escherichia coli. Infect. Immun., 3, 678.

ZWADYK, P. AND SNYDER, I. S. 1971. Purification and kinetic studies of the hemolysin from Escherichia coli. Can. J. Microbiol., 17, 741. 